\title{
Editorial Note on: The clinical study of repairing cauda equina fibres with fibrin glue after lumbar fracture and dislocation
}

Spinal Cord (2010) 48, 638; doi:10.1038/sc.2010.33; published online 27 April 2010

Editorial Note on: Spinal Cord (2010) 48, 633-637; doi:10.1038/sc.2009.195; published online 9 February 2010

This is a very interesting paper from Sun et al. ${ }^{1}$ Repairing injured nerve roots is a rarely reported topic. In a certain way, it can be compared with the repair of intracranial nerves, with regard to which it has been shown that repair of transacted nerves can be followed by a successful and, for a patient, useful recovery of the function. ${ }^{2}$

For lumbosacral nerve roots, the incidence of injury and specifically transaction is seldom seen. So far, only a handful of studies have described the functional recovery after such a trauma as demonstrated by Sun et al. ${ }^{1}$ in this study. Repair of the lumbosacral nerve roots can be worthwhile and some motor recovery can occur. The authors have chosen 'the right method' of repair using fibrin glue. This method of repair is the most atraumatic one for the nerve roots, as the glue provides additional stability, alignment and protection of the repaired nerve roots that is necessary for the regeneration process. More of such studies are welcome in order to stress the importance and potential of such a repair.

T Menovsky

Department of Neurosurgery, University Hospital of Antwerp, Antwerp, Belgium

E-mail: Tomas.menovsky@uza.be

\section{References}

1 Sun $\mathrm{T}, \mathrm{Lu} \mathrm{Z}$, Liu $\mathrm{S}, \mathrm{Xu} \mathrm{S}$. The clinical study of repairing cauda equina fibres with fibrin glue after lumbar fracture and dislocation. Spinal Cord 2010; 48: 633-637.

2 Menovsky T, van Overbeeke JJ. Intracranial nerve repair: a review of experimental and clinical studies. Neurosurg Focus 1996; 1: e2. 